\title{
BOOK-TAX DIFFERENCES E 0 GERENCIAMENTO DE RESULTADOS NAS EMPRESAS BRASILEIRAS DE CAPITAL ABERTO
}

\section{BOOK-TAX DIFFERENCES AND EARNINGS MANAGEMENT IN BRAZILIAN PUBLICLY TRADED COMPANIES}

“0 artigo foi aprovado e apresentado I Simpósio de Métodos Quantitativos, realizado de 08/11 a 09/11 de 2018, em Santa Maria (RS)"

\section{RESUIMO}

Este artigo teve por objetivo analisar a relação entre o gerenciamento de resultados e as book-tax differences (BTD) em uma amostra composta por 124 empresas brasileiras de capital aberto listadas na B3 com dados disponíveis no período de 2010 a 2015. Em termos metodológicos utilizou-se como variável dependente os accruals discricionários advindos do Modelo de Jones Modificado (1995) como proxy para gerenciamento de resultados, ao passo que a BTD, proxy para as diferenças entre o lucro contábil e o lucro tributável foi utilizada como variável explicativa. Adicionalmente foram incluídas no modelo duas variáveis de controle: i) alavancagem; e o ii) tamanho da firma. De modo geral, as evidências empíricas apontam a existência de uma relação diretamente proporcional entre o gerenciamento de resultados, a BTD, e o tamanho. Todavia, os resultados deste estudo não permitem confirmar a existência de uma relação significativa entre a alavancagem e o gerenciamento de resultados.

Palavras-chave: Gerenciamento de Resultados. Book-Tax Differences. Empresas abertas.

\begin{abstract}
This paper aimed to analyze the relationship between earnings management and book-tax differences (BTD) in a sample composed of 124 Brazilian companies listed in B3 with data available in the period from 2010 to 2015. In methodological terms, the discretionary accruals derived from the Modified Jones Model (1995) as a proxy for earnings management were used as the dependent variable, while the BTD, proxy for the differences between the accounting profit and the taxable profit was used as a variable explanatory. Additionally, two control variables were included in the model: i) leverage; and ii) size of the firm. In general, empirical evidence points to the existence of a directly proportional relationship between earnings management, BTD, and size. However, the results of this study do not confirm the existence of a significant relationship between financial leverage and earnings management.
\end{abstract}

Keywords: Earnings Management. Book-Tax Differences. Accruals. Publicly Traded Companies.
Paulo Fernando Marschner Mestre em Administração pela Universidade Federal de Santa Maria. Contato Av. Roraima, 1000 - 4303 - Cidade Universitária, Santa Maria - RS, 97105-900, E-mail: paulofernandomarschner@hotmail.com

\section{Vanessa Rabelo Dutra}

Doutoranda em Administração pela Universidade Federal de Santa Maria. Docente da Universidade Federal do Pampa. Contato Av. Roraima, 1000 - 4303 - Cidade Universitária, Santa Maria - RS, 97105-900, E-mail: vanessardg@gmail.com

\section{Kalú Schwaab}

Doutoranda em Administração pela Universidade Federal de Santa Maria. Contato Av. Roraima, 1000 - 4303 - Cidade Universitária, Santa Maria - RS, 97105 900, E-mail: kaluschwaab@gmail.com

\section{Paulo Sergio Ceretta}

Doutor em Engenharia de Produção pela Universidade Federal de Santa Catarina. Professor Associado da Universidade Federal de Santa Maria Contato Av. Roraima, 1000 - 4303 - Cidade Universitária, Santa Maria - RS, 97105 900, E-mail: ceretta10@gmail.com 


\section{INTRODUÇÃO}

A informação contábil em sua essência é um instrumento que fornece aos usuários conhecimentos quanto às condições da empresa (SCARPIN; PINTO; BOFF, 2007) e aos fenômenos contábeis que a envolvem (GONÇALVES et al. 2014). Ou seja, tem a função de reportar aos usuários a posição patrimonial fidedigna da entidade. A teoria da agência (JENSEN; MECKLING, 1976) tem amparado fortemente a contabilidade a compreender a importância de suas informações, pois por meio dessa teoria, sabe-se que apesar da existência dos contratos entre agente e principal, estes agem em prol da maximização da utilidade pessoal (NARDI; NAKAO, 2009).

Portanto, como não é possível abarcar todas as possíveis atitudes oportunistas que um agente pode tomar, e considerando a existência de assimetrias informacionais, as partes ficarão mais seguras quando bem informadas, e quando essas informações forem confiáveis e transparentes. Assim, quanto maior a qualidade nas informações menor tende a ser o nível de assimetrias informacionais, reduzindo a incerteza do usuário e acarretando decisões econômicas mais eficientes (NARDI; NAKAO, 2009). Sob esta ótica de análise, quando as informações contábeis fornecidas são de má qualidade, tendem ocasionar maiores custos de agência, originados pelos conflitos de interesse (DYCK; ZINGALES, 2004). As representações contábeis de baixa qualidade podem aumentar a demanda por mecanismos alternativos de monitoramento (PIRES, 2008). Por outro lado, quando fornecidas com qualidade, refletem-se favoravelmente nos custos de capital (LEUZ; VERRECCHIA, 2000), na assimetria informacional, na alocação de recursos e na eficiência de investimentos (BIDDLE; HILARY; VERDI, 2009).

Devido a necessidade de atender aos usuários das informações contábeis, as empresas tendem a manter dois conjuntos de demonstrações, de forma com que simultaneamente possam aumentar os lucros voltados ao mercado de capitais e diminuir os lucros reportados à tributação (DESAI, 2005). Em vista dessa realidade o gerenciamento de resultados e as divergências existentes entre a norma contábil e a tributária têm sido dois temas recorrentes na literatura contábil e de finanças. Tal perspectiva deve-se aos dois usuários associados a cada tema: os acionistas e o governo (HANLON; HEITZMAN, 2010; GRAHAM; RAEDY; SCHACKELFORD, 2012).

De acordo com Ferreira et al. (2012) o gerenciamento de resultados está associado às práticas discricionárias adotadas pelo gestor no intuito de manipular a informação contábil em consonância com algum tipo de incentivo econômico. As book-tax differences (BTD) estão basicamente relacionadas às diferenças existentes entre o lucro contábil e o lucro tributário, cabe ser destacado que o contraste existente entre os números contábeis e tributários pode ocorrer por dois principais motivos. Se por um lado pode ser ocasionado em função das normas que os orientam; por outro lado, pode decorrer a partir de incentivos e escolhas oportunistas, onde os gestores usam a discricionariedade sobre os números para atender a interesses (TANG, 2005).

Tais argumentos são confirmados empiricamente por Phillips, Pincus e Rego (2003) que indicaram uma melhoria nos modelos que detectam o gerenciamento de resultados contábeis quando utilizadas variáveis como proxy para a BTD. Nesse sentido, desenvolveu-se uma vasta literatura que procurou analisar a relação entre o gerenciamento de resultados e a BTD, os quais incluem os trabalhos de Mills e Newberry (2001), Tang (2005), Zimmerman e Goncharov (2006), Frank, Lynch e Rego (2009), Wilson (2009), Lang, Lins e Maffett (2012), Koubaa e Anis (2015) em âmbito internacional. E os trabalhos de Ferreira et al. (2012), Rezende e Nakao (2012) e Furtado, Souza e Neto (2016) em âmbito nacional.

Considerando a contextualização apresentada, o objetivo desta pesquisa é analisar a relação entre o gerenciamento de resultados e a BTD nas empresas brasileiras de capital aberto. Para operacionalização da pesquisa, utilizou-se como variável dependente os accruals discricionários advindos do Modelo de Jones Modificado (1995) como proxy para gerenciamento de resultados, ao passo que a BTD, proxy para as diferenças entre o lucro contábil e o lucro tributável foi utilizada como variável explicativa. Adicionalmente foram incluídas duas variáveis de controle: i) alavancagem; e o ii) tamanho da firma.

Embora exista um arcabouço teórico sobre o gerenciamento de resultados e as discrepâncias entre os números contábeis e tributários, a literatura nacional ainda necessita de investigações sobre essa relação. Diante de pouca evidência sobre este tema no mercado brasileiro, este trabalho contribui para o aprofundamento das discussões contábeis e de finanças proporcionando uma extensão ao conhecimento existente. 


\section{REFERENCIAL TEÓRICO}

\subsection{Gerenciamento De Resultados}

Embora os gestores ajam em conformidade com as exigências determinadas pelas normas contábeis no momento da elaboração das demonstrações, é preciso considerar que esses, em algumas circunstâncias, optam por uma alternativa em relação às demais que a norma contábil oferece (SPRENGER; KRONBAUER; COSTA, 2017). De acordo com Kronbauer (2008) as escolhas realizadas no momento da aplicação das normas contábeis criam um ambiente propício para que os gestores usem a discricionariedade para alterar os resultados.

A discricionariedade na forma de apresentação dos números contábeis pode ser ocasionada através do gerenciamento de resultados (FURTADO; SOUZA; NETO, 2016), que segundo Shipper (1989) trata-se de uma intervenção intencional no processo de elaboração das demonstrações contábeis, com a qual procura-se obter algum benefício particular. Tal intervenção torna-se difícil de ser evitada já que em diversas situações o gestor fica impossibilitado de obter regras para todos os fatos ocorridos (FIELDS; LYZ; VINCENT, 2001). Sendo assim, o gestor tem a oportunidade de fazer escolhas contábeis para alcançar determinado objetivo (HEALY, 1985).

A literatura existente apresenta diversas situações para a ocorrência do gerenciamento de resultados, as quais incluem: i) influenciar as percepções no mercado de ações, ii) aumentar a remuneração dos administradores, iii) reduzir a probabilidade de violar acordos de empréstimos, iv) evitar a intervenção regulamentar; v) evidenciar resultados positivos, vi) sustentar performance, e vii) atender às expectativas dos analistas (HEALY; WAHLEN, 1999; DEGEORGE; PATEL; ZECKHAUSER, 1999). O gerenciamento de resultados também pode ocorrer a partir do conflito de interesses entre o agente o e principal, como já emanado pela teoria da agência (JENSEN; MECKING, 1976). Tais conflitos podem resultar em assimetrias informacionais que podem prejudicar a qualidade dos lucros.

Embora todas as decisões tenham que ser aprovadas por um conselho, o gestor é o principal responsável pelas escolhas e, portanto, detém um conhecimento específico da firma em relação aos demais usuários, inclusive em relação aos acionistas (RONEN; YAARI, 2008). Devido a isso, é presumível que o gestor, de maneira oportunista, possa maximizar sua utilidade mesmo que isso prejudique a qualidade da informação divulgada, gerando assimetria informacional. Assim, entende-se que quanto maior o nível de gerenciamento de resultado apresentado pela firma, menor será a qualidade do lucro/prejuízo observado nas demonstrações contábeis (SUNDER, 1997).

Diante desse contexto, é possível observar que o gerenciamento de resultados pode ocorrer mediante a escolha de políticas contábeis ou por decisões operacionais (CARDOSO; MARTINEZ, 2009). A primeira vertente refere-se à parcela discricionária dos accruals, componentes que causam alteração no lucro/prejuízo em função do regime de competência (ALMEIDA, 2010). As decisões contábeis envolvem as escolhas das práticas contábeis relacionadas com: i) identificação do fenômeno - atos e fatos; ii) mensuração de seus efeitos no patrimônio da entidade; iii) classificação; iv) reconhecimento contábil; e v) sumarização e evidenciação da situação patrimonial e financeira da entidade.

Já a segunda ocorre mediante decisões operacionais e pode ser decorrente da motivação do gestor em iludir pelo menos alguns usuários das informações contábeis. Neste cenário, os gestores podem aumentar ou diminuir os lucros, os quais são gerenciados para atingir metas. Dessa forma, alguns usuários da informação poderão acreditar que determinadas metas foram atingidas (ROYCHOWDHURY, 2006). Um exemplo típico do gerenciamento mediante decisões operacionais decorre da implementação (ou não) de um desconto especial ou programa de incentivos para aumentar as vendas, próximo ao fim do trimestre no qual as metas de receita não foram atingidas. E por isso impactam o fluxo de caixa da entidade e, consequentemente, as receitas e as despesas associadas com essas atividades (MCKEE, 2005).

\subsection{Book-Tax Differences}

As book-tax differences (BTD) consistem nas diferenças que podem surgir entre o lucro contábil e o lucro tributável (HANLON; HEITZMAN, 2010), e podem ser ocasionadas devido à três circunstâncias: i) os diferentes objetivos entre as normas para a contabilidade financeira e a contabilidade tributária; ii) o gerenciamento de resultados contábeis; e iii) o gerenciamento tributário (CHAN; LIN; MO, 2010; HANLON; HEITZMAN, 2010). A primeira causa é o desalinhamento das normas que orientam a forma de cálculo do resultado financeiro e do resultado tributário (DESAI, 2005; FORMIGONI; ANTUNES; PAULO, 2009). As diferentes formas de cálculo, bem como os diversos usuários destas informações estimulam que algumas regras sejam aplicadas a um, ou a outro tipo de contabilidade.

As diferenças geradas por esse desalinhamento podem ser de dois tipos: permanentes e temporárias. As diferenças permanentes ocorrem quando determinadas receitas ou despesas são apuradas contabilmente, mas não possuem efeitos tributários. Já as diferenças temporárias ocorrem quando o tratamento para as normas contábeis e fiscais reconhecem o mesmo montante de receita ou despesa, e diferem apenas no período em que são reconhecidos, ou seja, contabilmente se considera um período, e fiscalmente outro (FORMIGONI; ANTUNES; PAULO, 2009; COMPRIX; GRAHAM; MOORE, 2011; SAYARI; MUGÃN, 2014).

A segunda causa é proveniente do gerenciamento de resultados contábeis e é utilizada pelos gestores a fim de evidenciar melhores resultados aos acionistas (MARQUES; COSTA; SILVA, 2016). Por fim, a terceira e última causa 
é o gerenciamento tributário e sua principal incidência é a motivação dos gestores em reduzir o montante a ser pago em impostos e taxas ao governo (HANLON; HEITZMAN, 2010). Portanto, a BTD pode ser composta de dois fatores: i) diferenças normais resultantes do desalinhamento dos conjuntos de regras contábeis e tributários; e ii) diferenças anormais ocasionadas por práticas discricionárias realizadas pelos gestores, ou seja, práticas de gerenciamento sobre o lucro contábil e/ou práticas de gerenciamento sobre o lucro tributável (MILLS; NEWBERRY, 2001).

\subsection{Trabalhos relacionados e desenvolvimento de hipóteses}

De acordo com Koubaa e Anis (2015) os gestores são frequentemente estimulados a usar as práticas de gerenciamento de resultados para aumentar os lucros contábeis, ao mesmo tempo em que visam reduzir a carga tributária. Sendo assim, tal manipulação pode ocasionar um aumento das diferenças entre o lucro contábil e o lucro tributário (BTD). Tang (2005) demonstrou em seu estudo uma associação positiva entre a BTD e o gerenciamento contábil e/ou de tributos no mercado chinês, no período de 1999 a 2004. Em uma perspectiva semelhante, Ferreira et al. (2012) encontraram evidências de um efeito positivo da BTD sobre valor dos accruals discricionários (proxy para o gerenciamento de resultados) em 118 empresas brasileiras de capital aberto.

Em um estudo multipaís, Lang, Lins e Maffett (2012) encontraram evidências de que a manutenção das diferenças entre o lucro contábil e o tributário afetam negativamente o nível de gerenciamentos de resultados. Rezende e Nakao (2012) encontraram uma relação negativa entre os incentivos econômicos vinculados à tributação e o nível de gerenciamento de resultados das empresas brasileiras de capital aberto de 1999 a 2007. Indicando, que quanto maior a diferença entre o lucro contábil e o tributário, menor será a utilização de escolhas discricionárias pelos gestores. Outros trabalhos que encontraram uma relação negativa incluem o de Zimmermann e Goncharov (2006) nas empresas russas de capital aberto de 2001 a 2002, e o de Furtado, Souza e Neto (2016) em empresas brasileiras de capital aberto. Devido às características de cada trabalho, não há um consenso sobre a relação entre o gerenciamento de resultados e a BTD, o que sugere a necessidade de ampliar a discussão sobre o tema. Desse modo, com o propósito de analisar essa relação, levantou-se a seguinte hipótese:

H1: Existe uma relação entre a BTD e o gerenciamento de resultado nas empresas brasileiras de capital aberto.

A alavancagem é uma variável utilizada em diversas pesquisas internacionais como controle para o gerenciamento de resultados (HSU, 2004; GU; LEE; ROSETT, 2005; MORSFIELD; TAN, 2006). De acordo com Hsu (2004) e Morsfield e Tan (2006) empresas com maior grau de alavancagem são estimuladas a manipular os resultados contábeis por diversos motivos, os quais incluem: i) aumentar seus resultados, ii) diminuir as restrições de empréstimo, e iii) contornar problemas com cláusulas de inadimplência. Ou seja, os gestores visando a continuidade da empresa e dos contratos com seus credores fazem uso de artifícios contábeis para manipular as informações de suas demonstrações (DEFOND; JIAMBALVO, 1994).

Por outro lado, o gerenciamento de resultados pode ocorrer com o objetivo de evidenciar uma redução nos ganhos, tendo em vista que investidores e credores de empresas mais endividadas tendem a exercer um monitoramento de forma mais ativa sobre a empresa (WATTS; ZIMMERMAN, 1986; BEATTY; WEBER, 2003). Devido à essas circunstâncias foi desenvolvida a seguinte hipótese:

H2: Existe uma relação entre a alavancagem e o gerenciamento de resultados nas empresas brasileiras de capital aberto.

O tamanho também é considerado um fator relacionado com o gerenciamento de resultados, pois de acordo com Hochberg (2004), a complexidade das demonstrações financeiras das empresas está intimamente relacionada ao tamanho da firma. Sendo assim, maiores serão os incentivos motivadores para que os gestores manipulem seus resultados. Para Gu, Lee e Rosett (2005) essa relação pode ser negativa devido a três fatores: i) as grandes empresas têm mais benefícios relativos à economia de escala; ii) são mais maduras e operam em um estado mais estacionário, e iii) tendem a ser mais diversificadas. Todas estas características levam a uma menor volatilidade operacional e, consequentemente, a uma menor variabilidade de accruals (TENDELOO; VANSTRAELEN, 2005). De acordo com essas evidências, pode-se esperar que o tamanho exerça um efeito positivo ou negativo sobre o gerenciamento de resultados. Devido a essa dualidade, têm-se a seguinte hipótese:

H3: Existe uma relação entre o tamanho e o gerenciamento de resultados nas empresas brasileiras de capital aberto.

Para analisar a relação entre o gerenciamento de resultados e as book-tax differences, foram delineados os procedimentos metodológicos a seguir.

\section{MÉTODO}

Para a formação da amostra, foram consideradas as empresas registradas como sociedades anônimas de capital aberto listadas na B3 S.A - Brasil, Bolsa, Balcão (excluindo as empresas financeiras), no período de 2010 a 2015, o qual 
foi definido por ser posterior a adoção das International Financial Reporting Standards (IFRS). Na amostra foram excluídos os dados de 2010, uma vez que esse período foi utilizado apenas para cálculo de defasagem de variáveis. Os dados foram coletados no banco de dados da Economática e a matriz foi composta de dados balanceados, ou seja, foram excluídas as empresas com dados inexistentes para todo o período. A amostra final foi composta por 124 empresas, que representaram um total de 620 observações.

\subsection{Modelo para Detecção do Gerenciamento de Resultados}

Para a mensuração dos accruals discricionários (proxy para o gerenciamento de resultados) utilizou-se o modelo proposto por Dechow, Sloan e Sweeney (1995). Este modelo retira do modelo de Jones (1991) a parcela da variação de contas a receber das variações de vendas, considerando assim a possibilidade de manipulação nas vendas a prazo. Nesta pesquisa, foi adotada a classificação dos accruals totais com base na variação das contas patrimoniais como variável dependente do modelo de Jones modificado (1995), como segue:

$$
T A_{i t}=\frac{\left(\Delta A C_{i t}-\Delta D i s p_{i t}\right)-\left(\Delta P C_{i t}-\Delta D I V_{i t}\right)-\text { DEpre }_{i t}}{A_{i t-1}}
$$

Onde, $T A_{i t}=$ accruals total da empresa $i$ no período $t, \Delta A C_{i t}=$ variação do ativo circulante da firma $i$ no período $t-1$ para o periodo $t ; \Delta$ Disp $p_{i t}$ $=$ variação das disponibilidades da firma $i$ no período $t$-1 para o período $t, \Delta P C_{i t}=$ variação do passivo circulante da firma $i$ no período $t-1$ para o período $t, \Delta D I V_{i t}=$ variação dos financiamentos e empréstimos de curto prazo da firma $i$ no período $t-1$ para o período $t ; \Delta D E p r e e_{i t}$ $=$ total das despesas com depreciação e amortização da firma $i$ no período $t, A_{i t-1}=$ total de ativos da empresa i defasado.

Após a mensuração dos accruals totais, utiliza-se o modelo de Jones modificado (1995) para determinar os accruals não discricionários que são calculados da seguinte forma:

$$
\frac{T A_{i t}}{A_{i t-1}}=\alpha_{1}\left(\frac{1}{A_{i t-1}}\right)+\alpha_{2}\left(\frac{\Delta R_{i t}-\Delta C R_{i t}}{A_{i t-1}}\right)+\alpha_{3}\left(\frac{P P E_{i t}}{A_{i t-1}}\right)+e_{i t}
$$

Onde, $T A_{i t}=$ accruals total da empresa $i$ no período $t ; A_{i t-1}=$ total de ativos da empresa $i$ defasado; $\Delta R_{i t}=$ variação da receita líquida da firma $i$ no período $t, \Delta C R_{i t}=$ variação de clientes da firma $i$ no período $t, \mathrm{PPE}_{i t}=$ imobilizado e intangível (brutos) da firma $i$ no período $t, e_{i t}$ é o resíduo da regressão.

Por meio de seu modelo, Dechow, Sloan e Sweeney (1995) separaram a parte não discricionária e explicaram que os accruals discricionários são identificados pelo próprio resíduo da regressão $\left(e_{i t}\right)$. Sendo assim os accruals discricionários terão como base de cálculo a diferença entre os totais e os não discricionários.

\subsection{Variáveis da Pesquisa, Tratamento e Análise dos Dados}

Durante a operacionalização do Modelo Jones Modificado, foram mensurados os accruals discricionários para toda a amostra, sendo obtidos por empresa-ano, assim como nos trabalhos de Ferreira et al. (2012), Furtado et al. (2016) e Sprenger et al. (2017). Os accruals discricionários são considerados proxy do gerenciamento de resultados, que corresponde a variável dependente. A Tabela 1 apresenta a BTD, o sinal esperado de acordo com a literatura e os autores que sustentam a relação, e as demais variáveis de controle.

Tabela 1 - Variáveis independentes, sinais esperados e autores

\begin{tabular}{c|c|c|l}
\hline Variável & Medida & Sinal Esperado & \multicolumn{1}{c}{ Autores } \\
\hline Book-tax differences & BTD $=\frac{\left(L A I R_{t}-L T_{t}\right)}{A T_{t-1}}$ & $(+)$ & $\begin{array}{l}\text { Tang (2005); Ferreira et al. (2012); Koubaa e } \\
\text { Anis, (2015); } \\
\text { Zimmerman e Goncharov (2006); Lang, Lins e } \\
\text { Maffett (201); Rezende e Nakao (2012); Furtado, } \\
\text { Souza e Neto (2016). } \\
\text { Defond e Jiambalvo (1994); Hsu (2004); Morsfield } \\
\text { e Tan (2006). } \\
\text { Watts e Zimmerman (1986); Beatty e Weber } \\
\text { (2003). }\end{array}$ \\
\hline
\end{tabular}




\begin{tabular}{c|c|c|l}
\hline Variável & \multicolumn{1}{|c|}{ Medida } & Sinal Esperado & \multicolumn{1}{c}{ Autores } \\
\hline Tamanho & $T A M=\ln ^{*}($ ATit $)$ & $(+)$ & $\begin{array}{l}\text { Hochberg (2004). } \\
\text { Gu, Lee e Rosett (2005); Tendeloo e Vanstraelen } \\
(2005) .\end{array}$ \\
\hline
\end{tabular}

Nota: Book-tax differences (BTD) é a diferença total entre o lucro contábil antes do imposto de renda (LAIR) e o lucro tributário (LT); AT é ativo total. O LT é resultado da divisão da despesa de imposto de renda pela alíquota fiscal máxima (34\%); Alavancagem (ALAV) é o passivo circulante (PC) mais o passivo não circulante (PNC), dividido pelo ativo total (AT); Tamanho (TAM) que é o logaritmo natural do ativo total (AT).

Fonte: Elaborado pelos autores (2018).

A variável dependente, assim como as independentes foram winsorizadas a $5 \%$, a fim de corrigir possíveis observações discrepantes (outliers), amenizando o desvio-padrão das variáveis e sua dispersão em torno da média. Dessa forma, antes de proceder as estimações e inferências estatísticas foram controlados os efeitos extremos para cada uma das variáveis. A análise dos dados está dividida em três etapas. A primeira consiste na aplicação da estatística descritiva nas variáveis utilizadas, a segunda a análise de correlação. A terceira procura verificar o impacto da BTD no gerenciamento de resultados, e para tal, foi proposto o modelo de regressão linear múltipla a seguir:

$$
D A(A B S)_{i t}=\alpha+\beta_{1} B T D_{i t}+\beta_{2} A L A V_{i t}+\beta_{3} T A M_{i t}+e_{i t}
$$

Onde: $D A(A B S)_{i t}=$ accruals discricionários na forma absoluta da empresa $i$ no período $t, B T D_{i t}=$ diferença entre o lucro contábil e o lucro tributável da empresa $i$ no período $t, A L A V_{i t}=$ alavancagem da empresa $i$ no período $t, T A M_{i t}=$ logaritmo natural da variável ativo total da empresa $i$ no período $t$; é o resíduo da regressão.

Existem basicamente três formas de simplificar e ajustar o modelo geral a fim de torná-lo mais funcional: o Modelo Pooled, o Fixed-Effects Model (Modelos Fixos) e o Random Effects (Efeitos Aleatórios). No primeiro modelo o intercepto é o mesmo para toda a amostra, ou seja, assume-se que todos os seus elementos possuem comportamento idêntico. O Modelo Pooled não considera o efeito do tempo, tão pouco o efeito individual de cada empresa (BALTAGI, 2001). O Modelo de Efeitos Fixos baseia-se na premissa de que os coeficientes da regressão podem variar de indivíduo para indivíduo ou no tempo, ainda que permaneçam como variáveis fixas, ou seja, não aleatórias (MARQUES, 2000). Este tipo de modelo pode, adicionalmente, ser dinâmico quando uma variável defasada é incluída no modelo, e estático, caso contrário (BALTAGI, 2001).

Por fim, o Modelo de Efeitos Aleatórios segue a premissa de que a influência do comportamento do indivíduo ou o efeito do tempo não podem ser conhecidos. Dessa forma, admite-se a existência do erro não correlacionado com os regressores. Marques (2000) afirma que a pressuposição que permeia o modelo é a de que o comportamento do indivíduo e do tempo não pode ser observado nem medido, e que em grandes amostras esse desconhecimento pode ser representado por uma variável aleatória normal, ou seja, o erro.

A escolha do modelo mais adequado para a amostra em estudo acontece mediante comparação realizada pelos seguintes testes: 1) Teste F, que compara a regressão Pooled com o Modelo de Efeitos Fixos; 2) Teste de Breusch-Pagan, que compara a regressão Pooled com o Modelo de Efeitos Aleatórios; e 3) Teste de Hausman, que verifica qual modelo, Fixo ou Aleatório, é o mais adequado.

\section{ANÁLISE E DISCUSSÃO DOS RESULTADOS}

Inicialmente na Tabela 2 são apresentadas as estatísticas descritivas das quatro variáveis analisadas. É possível observar que todas as variáveis possuem médias e medianas muito próximas. Os valores associados à assimetria indicam que as variáveis apresentam uma distribuição assimétrica, algumas concentradas à esquerda e outras à direita da média. Já os valores associados à curtose indicam que as variáveis accruals discricionários e tamanho apresentam uma distribuição platicúrtica já que seu valor é inferior a 3, valor de referência para uma distribuição normal. As variáveis BTD e alavancagem, por sua vez, apresentam uma leve distribuição leptocúrtica.

Pode-se observar ainda que a variável BTD apresentou uma oscilação distinta das demais, embora tenha apresentado uma média de $(0,012)$ os valores de mínimo e máximo indicam que algumas empresas no período tiveram uma BTD negativa $(-0,215)$ enquanto outras tiveram valores inversamente proporcionais $(0,182)$. A comparar as estatísticas descritivas com o trabalho de Ferreira et al. (2012), por exemplo, é possível observar uma leve redução nos valores da média, mediana, mínimo e máximo nas principais variáveis de interesse (Accruals discricionários e BTD), embora o desvio padrão tenha apresentado valores muito próximos. Isso pode estar associado ao diferente número de empresas em cada amostra, bem como ao intervalo temporal, que é posterior à adoção das IFRS. 
Tabela 2 - Estatística descritiva das variáveis das 124 empresas (período de 2011 a 2015, dados anuais).

\begin{tabular}{c|c|c|c|c}
\hline Estatistica & Book-tax diferences & Alavancagem & $\begin{array}{c}\text { Accruals } \\
\text { discricionários }\end{array}$ & Tamanho \\
\hline Média & 0,012 & 0,637 & 0,034 & 14,817 \\
Mediana & 0,017 & 0,607 & 0,032 & 14,854 \\
p10 & $-0,119$ & 0,371 & 0,006 & 12,746 \\
p25 & $-0,037$ & 0,480 & 0,017 & 13,640 \\
p75 & 0,072 & 0,744 & 0,046 & 16,941 \\
p90 & 0,144 & 0,921 & 0,070 & 2,544 \\
Variância & 0,009 & 0,054 & 0,005 & 10,195 \\
Mínimo & $-0,215$ & 0,267 & 0,003 & 18,964 \\
Máximo & 0,182 & 1,251 & 0,087 & 1,595 \\
Desvio Padrão & 0,098 & 0,233 & 0,023 & 0,017 \\
Assimetria & $-0,441$ & 0,872 & 0,675 & 2,691 \\
Curtose & 3,018 & 3,801 & 2,690 & \\
\hline
\end{tabular}

Fonte: Dados da pesquisa (2018).

Para a identificação das primeiras evidências da relação entre os accruals discricionários e a BTD, bem como para identificar os riscos de multicolinearidade, a Tabela 3 apresenta os coeficientes da correlação de Pearson entre as variáveis do modelo. A análise univariada revela uma associação positiva e significativa entre a variável dependente accruals discricionários e a variável BTD. Observa-se ainda, que estas não são altamente correlacionadas, indicando ausência de vieses na estimativa dos coeficientes. Ou seja, nenhuma variável produz um fator de inflação da variância (FIV) maior ou igual a 1, dessa forma, a multicolinearidade não será um problema para a estimativa da equação de regressão.

Tabela 3 - Correlação de Pearson

\begin{tabular}{l|c|c|c|c}
\hline Variáveis & Accruals discricionários & Book-tax differences & Alavancagem & Tamanho \\
\hline Accruals discricionários & 1 & & \\
Book-tax differences & $0,162^{\star \star \star}$ & 1 & \\
Alavancagem & $0,009^{\star}$ & $-0,652^{\star \star \star}$ & 1 & \\
Tamanho & $-0,021^{\star}$ & $0,158^{\star \star \star}$ & $-0,160^{\star \star \star}$ & 1 \\
\hline
\end{tabular}

Fonte: Dados da pesquisa (2018).

Nota: ${ }^{*}{ }^{* \star},{ }^{* \star *}$ estatisticamente significantes a $10 \%, 5 \%$ e $1 \%$, respectivamente

Para definição do modelo de regressão, foram comparadas as estimativas do Modelo Pooled com o Modelo de Efeitos Fixos através do Teste F. Um p-valor baixo contraria a hipótese nula da adequação do modelo Pooled, validando a hipótese alternativa da existência de Efeitos Fixos. De acordo com o Teste de Chow, o Modelo de Efeitos Fixos é preferido ao Modelo Pooled, tendo em vista o resultado: $F(123,493)=40,37$ (estatística calculada) com p-valor 0,000.

Posteriormente, para verificar se o Modelo Pooled é mais adequado do que o Modelo de Efeitos Aleatórios, foi utilizado o Teste de Breusch-Pagan. Como resultado, obteve-se LM = 931,11 com p-valor = prob (qui-quadrado $(1)>931,11$ ) $=0,000$. Um p-valor baixo contraria a hipótese nula de que o Modelo Pooled é adequado, validando a hipótese alternativa da existência de Efeitos Aleatórios. Neste caso, o Modelo de Efeitos Aleatórios deve ser preferido. Ao final, utilizou-se o Teste de Hausman para a escolha entre o Modelo Fixo e o Modelo Aleatório. Um p-valor baixo contraria a hipótese nula de que o Modelo de Efeitos Aleatórios é consistente, validando a hipótese alternativa da existência do Modelo de Efeitos Fixos. Tendo-se um $\mathrm{H}=30,14$ com p-valor $=0,000$, confirma-se a preferência pelo Modelo de Efeitos fixos, pois se rejeitou a hipótese nula lançada no referido teste.

Após esta etapa foi estimada a regressão e constatado que as variáveis BTD e Tamanho apresentam-se estatisticamente significantes na explicação dos accruals discricionários. A variável Alavancagem, por sua vez, não possui significância no modelo. 
Tabela 4 - Estimativas para o modelo de regressão com dados em painel (período de 2011 a 2015, dados anuais).

\begin{tabular}{|c|c|c|c|c|c|c|}
\hline $\begin{array}{c}\text { Accruals } \\
\text { discricionários }\end{array}$ & Coeficiente & $\begin{array}{c}\text { Erro } \\
\text { Padrão }\end{array}$ & $\mathbf{Z}$ & $p$-valor & \multicolumn{2}{|c|}{$\begin{array}{c}\text { [95\% intervalo de } \\
\text { confiança] }\end{array}$} \\
\hline Book-tax differences & 0,017 & 0,007 & 2,49 & 0,013 & 0,003 & 0,031 \\
\hline Alavancagem & $-0,003$ & 0,004 & $-0,80$ & 0,421 & $-0,011$ & 0,005 \\
\hline Tamanho & 0,002 & 0,004 & 2,42 & 0,015 & 0,000 & 0,004 \\
\hline Constante & 0,001 & 0,013 & 0,07 & 0,942 & $-0,023$ & 0,031 \\
\hline \multicolumn{7}{|c|}{ Ajustamento do modelo } \\
\hline Observações & 620 & \multicolumn{3}{|c|}{ Testes } & Coeficiente & p-valor \\
\hline $\mathrm{R} 2$ & 0,914 & \multicolumn{3}{|c|}{ Teste Jarque-Bera } & 11,98 & 0,002 \\
\hline R2-Ajustado & 0,054 & \multicolumn{3}{|c|}{ Teste Wald } & 4,05 & 0,000 \\
\hline F-statistic & 9,480 & \multicolumn{3}{|c|}{ Teste Wooldridge } & 23,10 & 0,000 \\
\hline Prob(F-statistic) & 0,000 & & & & & \\
\hline
\end{tabular}

Fonte: Dados da pesquisa (2018).

Ao observar os aspectos relacionados ao ajustamento do modelo, é possível verificar por meio do teste de normalidade Jarque-Bera (JB) que a distribuição dos resíduos é não normal. Verificou-se ainda a autocorrelação dos resíduos e a homocedasticidade dos dados em painel pelos Testes de Wooldridge e Wald, respectivamente. Nesse contexto, tais resultados sugeriram a autocorrelação serial dos resíduos e que os dados são heterocedásticos. Por meio desses resultados verifica-se que o modelo apresenta limitação de heterocedasticidade, autocorrelação serial e de não normalidade dos resíduos. Todavia, o teorema central do limite indica que os estimadores do método de mínimos quadrados ordinários tentem a satisfazer a normalidade assintótica aproximando-se de uma distribuição normal (WOOLDRIDGE, 2005). Dessa forma, mesmo com a existência de um teste específico, o pressuposto de normalidade é relaxado, pois seus coeficientes estimados são consistentes e não viesados assintoticamente, mesmo com presença de heterocedasticidade e autocorrelação (FORMIGONI; ANTUNES; PAULO, 2009).

É possível observar a partir da Tabela 4 que a BTD impacta positivamente os accruals discricionários, indicando que quanto maior a diferença entre o lucro contábil e o lucro tributável, maior será o montante das acumulações discricionárias em sua forma absoluta. Tal constatação é suportada por Tang (2005), Ferreira et al. (2012) e Koubaa e Anis (2015) os quais encontraram evidências de que quanto maior o valor da BTD, maior o valor dos accruals discricionários, sugerindo que esta variável pode servir de suplemento para detectar práticas discricionárias utilizadas pelos gestores. Esse resultado suporta a premissa de que os gestores podem usar as práticas de gerenciamento de resultados para aumentar os lucros contábeis, ao mesmo tempo em que visam reduzir a carga tributária. A partir desse resultado é possível validar a hipótese H1 de que existe uma relação entre a BTD e o gerenciamento de resultados nas empresas brasileiras de capital aberto.

A variável alavancagem não apresenta uma relação significativa no modelo, o que se opõe aos trabalhos de Watts e Zimmerman (1986), Defond e Jiambalvo (1994), Beatty e Weber (2003), Hsu (2004) e Morsfield e Tan (2006) que indicaram uma relação significativa entre as variáveis. Todavia, a evidência de uma relação não significativa é semelhante a encontrada por Ardison, Martinez e Galdi (2008) nas empresas brasileiras. A partir desse resultado não é possível confirmar a hipótese $\mathrm{H} 2$ de que existe uma relação entre alavancagem e o gerenciamento de resultados nas empresas brasileiras de capital aberto.

A variável tamanho apresentou um coeficiente positivo e significativo, que embora pequeno, sugere que o tamanho da firma impacta positivamente os accruals discricionários. Este resultado se opõe aos resultados de Gu, Lee e Rosett (2005) e Tendeloo e Vanstraelen (2005) que indicaram um efeito negativo. A evidência de um efeito positivo está de acordo com Hochberg (2004) que sustenta essa relação e efeito atrelando a complexidade das demonstrações financeiras ao seu tamanho. A partir desse resultado é possível aceitar a hipótese H3 de que existe uma relação entre o tamanho e o gerenciamento de resultados nas empresas brasileiras de capital aberto.

De modo geral, as evidências empíricas apontam a existência de uma relação diretamente proporcional entre o gerenciamento de resultados, as diferenças entre o lucro contábil e o tributário (BTD), e o tamanho da firma. Tais evidências suportaram parcialmente a vertente teórica que balizam as hipóteses $\mathrm{H} 1 \mathrm{e} \mathrm{H} 3$. Contudo, os resultados deste estudo não permitem confirmar a existência de relação entre alavancagem e o gerenciamento de resultados, conforme esperado por parte da literatura.

\section{CONSIDERAÇÕES FINAIS}

O objetivo deste trabalho foi analisar a relação entre o gerenciamento de resultados e as book-tax differences nas empresas brasileiras de capital aberto. A amostra da pesquisa foi composta por 124 companhias listadas na B3 S.A - Brasil, Bolsa, Balcão com dados disponíveis para o período de 2010 a 2015. 
Tang (2006), Ferreira et al. (2012) e Koubaa e Anis (2015) sugerem uma relação positiva entre a BTD e o gerenciamento de resultados. Por outro lado, Zimmerman e Goncharov (2006), Lang, Lins e Maffett (2011), Rezende e Nakao (2012) e Furtado, Souza e Neto (2016) sugerem uma relação negativa. Os resultados desta pesquisa evidenciaram que as variáveis possuem relação significativa e positiva, o que sustenta empiricamente a hipótese H1 de que a diferença entre o lucro contábil e o lucro tributável (BTD) possui uma relação com o gerenciamento de resultado nas empresas brasileiras de capital aberto, e atende ao objetivo geral do trabalho. Esse resultado pode ser decorrente do uso das práticas de gerenciamento visando aumentar os lucros contábeis, ao mesmo tempo em que visam reduzir a carga tributária, como destacado por Koubaa e Anis (2015).

Na variável alavancagem, parte dos trabalhos sugeriam um relação positiva (DEFOND; JIAMBALVO, 1994; HSU, 2004; MORSFIELD; TAN, 2006), e outra parte um relação negativa (WATTS; ZIMMERMAN, 1986; BEATTY; WEBER, 2003), o que não foi confirmado rejeitando a Hipótese H2. A hipótese H3, suportada por Gu, Lee e Rosett (2005) e Tendeloo e Vanstraelen (2005) e Hochberg (2004) sugere uma relação significativa entre o tamanho e o gerenciamento de resultados, o que foi confirmado, não rejeitando a hipótese H3. Ou seja, nas empresas abertas brasileiras a complexidade das demonstrações financeiras podem estar intimamente relacionadas ao tamanho da firma a tal ponto que serve de estímulo para que os gestores manipulem seus resultados (HOCHBER, 2004).

Em síntese, estes resultados permitiram expandir os achados empíricos sobre a relação entre o gerenciamento de resultados e BTD. De posse de tais evidências, percebe-se que a utilidade das informações contidas nas diferenças entre o lucro contábil e o lucro tributável são de suma importância para os diversos usuários da informação contábil, já que podem ser usadas para informar não só as autoridades fiscais, mas também os investidores sobre a situação financeiras das empresas. Além disso, os resultados demonstram que a BTD pode servir de complemento para detectar práticas discricionárias utilizadas pelos gestores, evidenciando, na prática os fundamentos da teoria da agência. Tendo conhecimento dessa complexidade, um entendimento detalhado de sua relação com o gerenciamento de resultados é salutar para uma análise da qualidade dos resultados atuais e na previsão de resultados futuros.

Adicionalmente, este estudo apresenta algumas limitações. Cumpre ressaltar que os achados deste trabalho restringem-se às empresas analisadas, bem como ao intervalo de tempo definido. Outra limitação foi a impossibilidade de se fazer inferências sobre os resultados referentes a alavancagem. Por se tratar de uma literatura recente no Brasil, sugere-se um aprofundamento do tema, por meio de pesquisas futuras. Possíveis extensões poderiam incluir uma análise da BTD por tipo (temporária e permanente), bem como outras formas de medir o gerenciamento de resultados.

\section{REFERÊNCIAS}

ALMEIDA, J. E. F. Qualidade da informação contábil em ambientes competitivos. 2010. Tese (Doutorado em Controladoria e Contabilidade) - Programa de Pós-Graduação em Ciências Contábeis, Faculdade de Administração, Economia e Contabilidade da Universidade de São Paulo. São Paulo, 2010.

ARDISON, K. M. M.; MARTINEZ, A. L.; GALDI, F. C. The effect of leverage on earnings management in Brazil. Advances in Scientific and Applied Accounting, v. 5, n. 3, p. 305-324, 2012

BALTAGI, B. H. Econometric analysis of panel data. West Sussex, UK: John Wiley, 2001.

BEATTY, A.; WEBER, J. The effects of debt contracting on voluntary accounting method changes. The Accounting Review, v.76, p. $27-59,2003$

BIDLLE, G. C.; HILARY, G.; VERDI, R. S. How does financial reporting quality relate to investment efficiency? Journal of Accounting and Economics, v. 48, n. 2, p. 112-131, 2009.

CARDOSO, R. L.; MARTINEZ, A. L. Gerenciamento da informação contábil no Brasil mediante decisões operacionais. Revista Eletrônica de Administração, v. 15, n. 3, p. 1-27, 2009.

CHAN, K. H.; LIN, K. Z.; MO, P. L. L. Will a departure from tax-based accounting encourage tax noncompliance? Archival evidence from a transition economy. Journal of Accounting and Economics, v. 50, n. 1, p. 58-73, 2010.

COMPRIX, J.; GRAHAM, R. C.; MOORE, J. A. Empirical Evidence on the Impact of Book-Tax Differences on Divergence of Opinion among Investors. Journal of the American Taxation Association, v. 33, n. 1, p. 51-78, 2011.

DECHOW, P. M.; SLOAN, R. G.; SWEENEY,. Detecting Earnings Management. The Accounting Review, v. 70, n. 2, p. 193-225, 1995.

DEFOND, M. L.; JIAMBALVO, J. Debt covenant violation and manipulation of accruals. Journal of Accounting and Economics, v. 17, n. 1, p. $145-176,1994$.

DEGEORGE, F.; PATEL, J.; ZECKHAUSER, R. Earnings management to exceed thresholds. Journal of Business, v. 72, p. 1-33, 1999.

DESAI, M. A. The Degradation of Reported Corporate Profits. Journal of Economics Perspectives, v. 19, n. 4, p. 171-192, 2005.

DYCK, A.; ZINGALES, L. Private benefits of control: An international comparison. The Journal of Finance, v. 59, n. 2, p. 537-600, 2004.

FERREIRA, F. R.; MARTINEZ, A. L.; COSTA, F. M.; PASSAMANI, R. R. Book-tax differences e o gerenciamento de resultados no mercado de ações do Brasil. Revista de Administração de Empresas, v. 52, n. 5, p. 488-501, 2012.

FIELDS, T. D.; LYZ, T. Z.; VINCENT, L. Empirical research on accounting choice. Journal of Accounting \& Economics, v. 31, p. 255-307, 2001. 
FORMIGONI, H.; ANTUNES, M. T. P.; PAULO, E. Diferença entre o lucro contábil e lucro tributável: uma análise sobre o earnings management contábeis e gerenciamento tributário nas companhias abertas brasileiras. Brazilian Business Review, v. 6, n. 1, p. 44-61, 2009.

FRANK, M. M.; LYNCH, L. J.; REGO, S. O. Tax Reporting Aggressive Ness and its Relation to Agressive Financial Reporting. The Accounting Review, v. 84, n. 2, p. 467-496, 2009.

FURTADO, L. L.; SOUZA, J. A. S.; NETO, A. S. Gerenciamento de resultados contábeis à luz das diferenças entre o lucro contábil e tributário (book-tax differences): uma análise de dados em painel balanceado. Revista Ambiente Contábil, v. 8, n. 1, p. 115-132, 2016.

GRAHAM, J. R.; RAEDY, J. S.; SCHACKELFORD, D. A. Research in accounting for income taxes. Journal of Accounting and Economics, v. 53, n. 1, p. 412-434, 2012.

GONÇALVES, J. C.; BATISTA, B. L. L.; MACEDO, M. A. S.; MARQUES, J. A. V. C. Análise do impacto do processo de convergência às normas internacionais de contabilidade no Brasil: um estudo com base na relevância da informação contábil. Revista Universo Contábil, v. 10, n. 3, p. 25-43, 2014.

GU, Z.; LEE, C. W. J.; ROSETT, J. G. What determines the variability of accounting accruals? Review of Quantitative Finance and Accounting, v. 24, p. 313-334, 2005.

HANLON, M.; HEITZMAN, S. A review of tax research. Journal of Accounting and Economics, v. 2, p. 127-178, 2010.

HEALY, P. M. The effect of bonus schemes of accounting decisions. Journal of Accounting and Economics, v. 7, p. 85-107, 1985.

HEALY, P. M.; WAHLEN, J. M. A Review of the Earnings Management Literature and Its Implications for Standard Setting. Accounting Horizons, v. 13, n. 4, p. 365-383, 1999.

HOCHBERG, Y. V. Venture capital and corporate governance in the newly public firm. Review of Finance, Fothcoming - San Diego, 2004

HSU, P. C. Going Private-A Response to an Increased Regulatory Burden? UCLA School of Law, Law-Econ Research Paper, p. 04-16, 2004

JENSEN, M. C.; MECKLING, W. H. Theory of the firm: Managerial behavior, agency costs and ownership structure. Journal of Financial Economics, v. 3, n. 4, p. 305-360, 1976.

JONES, J. J. Earnings Management During Import Relief Investigations. Journal of Accounting Research, v. 29, n. 2, p. 193-228, 1991.

KOUBAA, R. R.; ANIS,. Book-tax differences: relevant explanatory factors. International Journal of Accounting and Economics Studies, v. 3, n. 2, p. 95-104, 2015.

KRONBAUER, C. A. Información relativa al impuesto sobre beneficios: análisis empírico sobre la divulgación realizada por las empresas del IBEX-35 y del IBOVESPA en los ejercicios de 2003/2005. PhD Thesis. (Doutorado em Contabilidade e Auditoria). Departamento de Contabilidade e Economia. Universidade de Sevilla, Espanha, 2008.

LANG, ; LINS, K. V.; MAFFETT,. Transparency, liquidity, and valuation: International evidence on when transparency matters most. Journal of Accounting Research, v. 5, n. 3, p. 729-774, 2012.

LEUZ, C.; VERRECCHIA, R. E. The economic consequences of increased disclosure. Journal of Accounting Research, v. 38, n. 1, p. $91-124,2000$.

MCKEE, Thomas E. Earnings management: an executive perspective. Ohio: Thomson. 2005.

MARQUES, L. D. Modelos dinâmicos com dados em painel: revisão de literatura. Dissertação (Mestrado) - Faculdade de Economia do Porto, Portugal, 2000.

MARQUES, A. V. C.; COSTA, P. S.; SILVA, P. R. Relevância do Conteúdo Informacional das Book-Tax Differences para Previsão de Resultados Futuros: Evidências de Países-Membros da América Latina. Revista Contabilidade \& Finanças, v. 27, n. 70, p. 29-42, 2016.

MILLS, L.; NEWBERRY, J. The influence of tax and non-tax costs on book-tax reporting differences: Public and private firms. Journal of the American Taxation Association, v. 23, n. 1, p. 1-19, 2001.

MORSFIELD, S. G.; TAN, C. E. L. Do venture capitalists influence the decision to manage earnings in initial public offerings? Accounting Review, v. 81, n. 5, p. 1119-1150, 2006.

NARDI, P. C. C.;NAKAO, S. H. Gerenciamento de resultados e a relação com o custo da dívida das empresas brasileiras abertas. Revista Contabilidade \& Finanças, v. 20, n. 51, p. 77-100, 2009.

PHILLIPS, J.; PINCUS, K.; REGO, S. O. Earnings Management: New Evidence Based on Deferred Tax Expense. The Accounting Review, v. 78, n. 2, p. 491-521, 2003.

PIRES, Rodolpho, G.; A informação contábil e a teoria de agência: um estudo da assimetria informacional em companhias abertas, listadas no novo mercado da Bovespa. Dissertação de Mestrado. 2008.

REZENDE, G. P.; NAKAO, S. H. Gerenciamento de resultados e a relação com o lucro tributável das empresas brasileiras de capital aberto. Revista Universo Contábil, v. 8, n. 1, p. 6-21, 2012.

RONEN, J.; YAARI, V. Earnings Management Emerging Insights in Theory, Practice, and Research. New York: Springer, 2008.

ROYCHOWDHURY, S. Earnings management through real activities manipulation. Journal of Accounting and Economics, v. $\underline{42, \mathrm{n}}$. 3, p. 335-370, 2006

SAYARI, G.; MUGÃN, F. N. Comparison of Book Income and Taxable Income in Terms of Value Relevance of Earnings. World of Accounting Science, v. 16, n. 1, p. 1-21, 2014. 
SCARPIN, J. E.; BOFF, M. L.; PINTO, J. A relevância da informação contábil e o mercado de capitais: uma análise empírica das empresas listadas no índice Brasil. In: Congresso USP de Contabilidade e Controladoria, IX, 2007, São Paulo, SP. Anais... São Paulo: USP, 2007.

SHIPPER, K. Commentary: earnings manegement. Accounting Horizons, [s.I], 1989. 91-102.

SPRENGER, K. B.; KRONBAUER, C. A.; COSTA, C. M. Características do CEO e o gerenciamento de resultados nas empresas listadas na BM\&FBOVESPA. Revista Universo Contábil, v. 13, n. 3, p. 120-142, 2017.

SUNDER, S. Theory of accounting and control. [S.1.]: Cincinnati: South-Western Publishing, 1997.

TANG, T. Book-tax differences: a function of accounting-tax misalignment, earnings management and tax management: empirical evidence from China. In: American Accounting Association Annual Meeting, Washington DC, 2005.

TENDELOO, B. V.; VANSTRAELEN, A. Earnings management under German GAAP versus IFRS. European Accounting Review, v. 14, n. 1, p. 155-180, 2005.

WATTS, R. L.; ZIMMERMAN, J. L. Positive accounting theory. Englewood Cliffs: Prentice Hall, 1986.

WILSON, R. J. An examination of corporate tax shelter participants. The Accounting Review, v. 84, n. 3, p. 969-999, 2009.

WOOLDRIDGE, J. Introductory Econometrics. South-Western Publishing, 2005.

ZIMMERMANN, J.; GONCHAROV, I. Earnings management when incentives compete: the role of tax accounting in Russia. Journal of International Accounting Research, v. 5, n. 1, p. 41-65, 2006. 